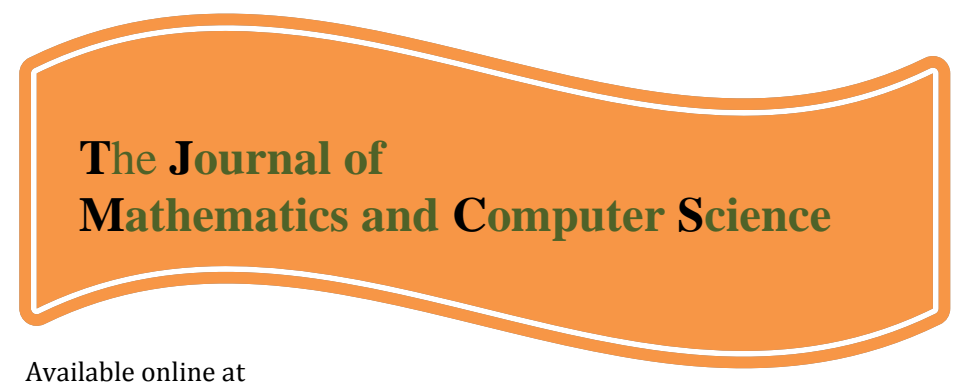

http://www.TJMCS.com

The Journal of Mathematics and Computer Science Vol .1 No.4 (2010) 321-332

\title{
Fuzzy Demand Consideration in a Multi-Objective Dynamic Cell Formation Problem Using a Robust Scatter Search
}

\author{
Hossein Amoozad-Khalili1 ${ }^{1,}$, Mehdi Ranjbar-Bourani'2, S.M.Javad Mirzapour Ale-Hashem ${ }^{3}$ \\ Faculty Member of Islamic Azad University-Nowshahr Branch, Nowshahr, Iran, amoozad_ie@iauns.ac.ir \\ Department of Industrial Engineering, I.A.U., South Tehran Branch, Tehran, Iran, ranjbar.mehdi@yahoo.com \\ Department of Industrial Engineering, Iran University of Science and Technology, Tehran, Iran, \\ b.mirzapour@gmail.com
}

Received: July 2010, Revised: October 2010

Online Publication: December 2010

\begin{abstract}
This paper presents a multi-objective cell formation problem considering alternative process routes and machine utilization with fuzzy demand. Two conflicting objectives include the total cell load variation and sum of the other costs consisting machine cost, inter-cell material handling cost, parts purchasing, operation, maintenance, and reconfiguration of machines cost are to be minimized simultaneously. Moreover, we consider demand in fuzzy condition, because it is more realistic to take into account the inexact and uncertain nature of demand. Due to the complexity of this problem, we develop a scatter search algorithm. Also by using the Taguchi as a robust parameter design method, we tune the effective factors of the developed algorithm on two sizes of benchmark problems that are generated randomly. NSGAII and Scatter Search evaluated and the related results confirm the efficiency and the effectiveness of our proposed Scatter Search provides good output according to some quality measures, especially for large-sized problems.
\end{abstract}

Keywords: Cell formation problem, fuzzy demand, scatter search, Taguchi design

\section{Introduction}

In many practical cases, a product mix or demand level may vary under a multi-period

\footnotetext{
1,* Corresponding author: MSc in Industrial Engineering

${ }^{2} \mathrm{MSc}$ in Industrial Engineering

${ }^{3} \mathrm{Ph} . \mathrm{D}$ Candidate in Industrial Engineering
} 
Table 1. Reasons for considering impreciseness in CMS design parameter.

\begin{tabular}{|c|ll|}
\hline CMS Design Parameter & & \multicolumn{1}{c|}{ Uncertainty Reason } \\
\hline & $\bullet$ & Time gap between design and implementation \\
& • & High cost in acquiring system parameters with precision \\
Part Demand & - & Insufficient market survey at design stage \\
& - & Product specifications not yet finalized \\
& - & Unknown product mix \\
& - & Competitor's competence and preparedness \\
\hline
\end{tabular}

planning horizon. A DCMS considers reconfiguration of cells in each period and brings flexibility to form machine cells and part families. Some investigations have been carried out in the field of CFPs under dynamic conditions by Vakharia and Kaku [1], Harhalaks et al. [2], Wilhelm et al. [3], and Askin et al. [4]. Chen [5] proposed a mixed-integer programming (MIP) model that minimizes the reconfiguration costs, machine constant costs, and intracellular movements. Balakrishnan and Cheng [6] also considered a two-step model for the generalized machine assignment problem and dynamic programming for the CFP with changeable part demands. Tavakkoli-Moghaddam et al. [7] developed the model, which was first proposed by Chen, with additional assumptions such as: alternative process plan, sequence operation, machine capacity and machine replication with aim of minimizing the sum of machine total costs and inter-cell movements cost simultaneously. Defersha and Chen [8] proposed a comprehensive mathematical model for a DCMS based on tooling requirements of the parts, tooling available on the machines, dynamic cell configuration, alternative routings, lot splitting, sequence of operations, multiple units of identical machines, machine capacity, operation cost, parts' outsourcing cost, tool consumption cost, setup cost, cell size limits, and machine adjacency constraints.

Safaei et al. [9] presented a mixed-integer programming model for a dynamic cell formation problem with fuzzy parameters, such as part demand and machine availability. Torabi and Hassini [10] presented a new multi-objective possibility mixed-integer linear programming model with some fuzzy parameters, such as market demands, cost/time coefficients and capacity levels.

Short life cycle, high variation manufacturing, unpredictable demand, and short lead-time have pushed production systems to operate dynamically under unreliable conditions [5]. Besides, marketing development takes the uncertain nature of the model parameters into consideration based on the fuzzy theory. Table 1 lists a number of reasons for considering uncertainty in CMS design parameter [11].

For dynamic and uncertain manufacturing requirements, it is necessary to identify different demands and mixtures of each part type per period through a known membership function. Seifoddini [12] considered uncertainty in form of probabilistic demands for a CFP, but under one period planning horizon. Tavakkoli-Moghaddam et al. [13] extended their previous model and considered trapezoid instead of triangular fuzzy numbers to show the demand uncertainty. They also modified the proposed mathematical model to a mixed-integer nonlinear programming (MINLP) model with fuzzy parameters [14]. This paper proposes an extended MINLP model with fuzzy Demand for the CFP considering two objectives: 1. minimizing the dynamic system total cost; 2 . minimizing the intracellular workload variation The main constraints are the cell size limitation, machine capacity, machine capability of processing an operation, machine investment, and production volume.

The rest of this paper is organized as follows. Detailed description of the proposed model is described in Section 2. Section 3 proposes the design of MOSS along with the Taguchi design for tuning the parameters of mentioned algorithm. A numerical example and the computational results are reported in Section 4. Discussion and conclusion are presented in Section 5. 


\section{Proposed mathematical model}

2.1. Notations The notations of the proposed model are described as follows:

$\begin{array}{ll}p: & \text { Part } \quad \text { types; } \\ & p=1,2, \ldots, P \\ j: & \text { Operations }\end{array}$
required by part

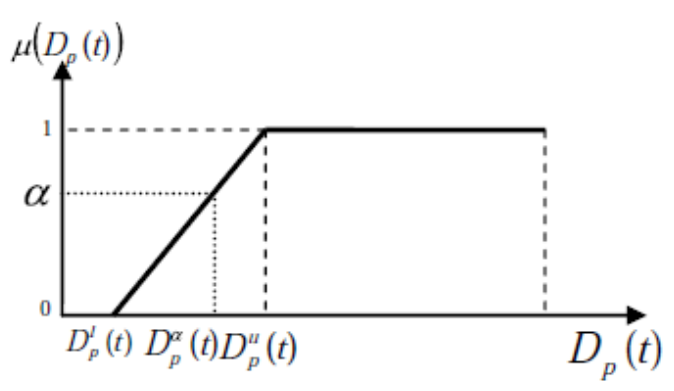

Figure 1. Membership function for a fuzzy demand of part $p$ in period $t$.

$$
p ; j=1,2, \ldots, J_{p}
$$

$m: \quad$ Machine types; $j=1,2, \ldots, J_{p}$

$c: \quad$ Manufacturing cells; $c=1,2, \ldots, C$

$t: \quad$ Time types; $t=1,2, \ldots, T$

$P$ : $\quad$ Number of part types.

$J_{p}: \quad$ Number of operations for part $p$.

$M: \quad$ Number of machine types.

$C: \quad$ Maximum number of cells that can be formed.

$T: \quad$ Number of manufacturing periods.

$\tilde{D}_{p}(t): \quad$ Fuzzy demand for part $p$ in period $t$ in form of a fuzzy number (see Figure 1) with a piecewise membership function (see Equation (1) [15].

$\mu\left(D_{p}(t)\right)= \begin{cases}0 & D_{p}(t) \leq D_{p}^{l}(t) \\ \frac{D_{p}(t)-D_{p}^{l}(t)}{D_{p}^{u}(t)-D_{p}^{l}(t)} & D_{p}^{l}(t)<D_{p}(t)<D_{p}^{u}(t) \\ 1 & D_{p}(t) \geq D_{p}^{u}(t)\end{cases}$

From the point of the DM, the demand of part $p$ in period $t$ should be presumably equal and greater than $D_{p}^{u}(t)$. In other words, $D_{p}^{u}(t)$ indicates a desirable level for part demand. Therefore, the interval $\left(D_{p}^{u}(t), \infty\right)$ presents a "risk-free" value-interval and $\left(D_{p}^{l}(t), D_{p}^{u}(t)\right)$ presents a "risk-full" value-interval for the decision maker. In the proposed model, there is a trade-off between maximizing the decision maker's utility and minimizing the sum of traditional costs of the CFP. Thus, interval $\left[D_{p}^{u}(t), \infty\right)$ is not considered in our model, because the production volume within this interval causes simultaneous increasing operation costs while the decision maker's utility remains constant.

$\alpha$ Cut-level This parameter is determined by the DM and used to convert the fuzzy proposed model into a crisp parametric model. The $\alpha$-level cut concept [16] limits the range of demand for part $p$ in period $t$ and capacity of machine type $m$ according to the DM's preferences. For any $\alpha$ value, we have an optimal solution; so the solution with $\alpha$ grade of membership is actually fuzzy [17].

$B_{p} \quad$ Batch size for inter-cell material movements for processing two consecutive operations of part type $p$.

$V_{p} \quad$ Inter-cell material movement cost for each batch of part type $p$.

$U_{p} \quad$ Subcontracting cost for each batch of part type $p$. 
$M_{m} \quad$ Maintenance cost of machine type $m$ (constant).

$O_{m} \quad$ Operating cost of machine type $m$ per hour (variable).

$h_{j p m} \quad$ Time required performing operation $j$ of part type $p$ on machine type $m$ (constant).

$\beta_{j p m}=\left\{\begin{array}{l}1 \text { if operation } j \text { of part type } p \text { can be done } \\ \text { on machine type } m \\ 0 \text { otherwise }\left(h_{j p m}=0\right)\end{array}\right.$

$r_{m} \quad$ Relocation cost of machine type $m$.

$U B \quad$ Upper bound for the cell size.

$P_{m}(t) \quad$ Purchasing cost of machine type $m$ in period $t$.

$Y_{m}(t) \quad$ Maximum allowed number of machine type $m$ to procure in period $t$.

$w_{j p m c}(t) \quad$ Workload on machine type $m$ in cell $c$ due to performing operation $j$ of part type $p$ in $\operatorname{period} t$.

$\bar{w}_{j p c}(t) \quad$ Average workload on each machine in cell $c$ due to performing operation $j$ of part type $p$ in period $t$.

\subsection{Decision Variables}

$N_{m c}(t) \quad$ Number of machine type $m$ in cell $c$ in period $t$.

$n_{m c}^{+}(t) \quad$ Number of increased machine type $m$ to cell $c$ in period $t$.

$n_{m c}^{-}(t) \quad$ Number of decreased machine type $m$ from cell $c$ in period $t$.

$Q_{p}(t) \quad$ Production volume of part type $p$ in period $t$.

$\varepsilon_{j p m c}(t) \quad$ The proportion of the total demand of part type $p$ with operation $j$ to perform by machine type $m$ in cell $c$ during period $t$.

$\delta_{p}(t) \quad$ The proportion of the total demand of part type $p$ to be subcontracted in period $t$.

$Z_{j p c}(t)=\left\{\begin{array}{l}1 \text { if operation } j \text { of part type } p \text { can be } \\ \text { done in cell } c \text { during period } t \\ 0 \text { otherwise }\end{array}\right.$

\subsection{Mathematical model}

Based on the definitions that describe in complete paper, the proposed model for the CFP under dynamic and fuzzy demand is illustrated as follows:

$$
\begin{aligned}
& \min Z_{1}=\sum_{t=1}^{T} \sum_{m=1}^{M} \sum_{c=1}^{C} N_{m c}(t) \cdot M_{m}+\sum_{t=1}^{T} \sum_{c=1}^{C} \sum_{m=1}^{M} \sum_{p=1}^{P} \sum_{j=1}^{J_{P}} Q_{P}(t) \cdot \varepsilon_{j p m c}(t) \cdot h_{j p m} \cdot o_{m}+\frac{1}{2} \sum_{t=1}^{T} \sum_{c=1}^{C} \\
& \sum_{m=1}^{M}\left(n_{m c}^{+}(t)+n_{m c}^{-}(t)\right) \cdot r_{m}+\sum_{t=2}^{T} \sum_{m=1}^{M} p_{m}(t) \cdot\left(\sum_{c=1}^{C} N_{m c}(t)-\sum_{c=1}^{C} N_{m c}(t-1)\right)+\frac{1}{2} \sum_{t=1}^{T} \sum_{p=1}^{P}\left[\frac{Q_{P}(t)}{B_{p}}\right] \cdot V_{p} \cdot\left(1-\delta_{p}(t)\right) \\
& \times \sum_{j=1}^{J_{P}-1} \sum_{c=1}^{C}\left|Z_{(j+1) p c}(t)-Z_{j p c}(t)\right|+\sum_{t=1}^{T} \sum_{p=1}^{P} Q_{P}(t) \cdot \delta_{p}(t) \cdot U_{P}
\end{aligned}
$$$$
\min Z_{2}=\sum_{t=1}^{T} \sum_{m=1}^{M} \sum_{c=1}^{C} \sum_{p=1}^{P} \sum_{j=1}^{J_{p}}\left|w_{j p m c}(t)-\bar{w}_{j p c}(t)\right|
$$ 


$$
\begin{aligned}
& \varepsilon_{j p m c} \leq \beta_{j p m} \quad \forall j, p, m, c, t \\
& \sum_{p=1}^{P} \sum_{j=1}^{J_{p}} Q_{P}(t) \cdot \varepsilon_{j p m c}(t) \cdot h_{j p m} \leq N_{m c}(t) \cdot C_{m} \quad \forall m, c, t \\
& \sum_{m=1}^{M} N_{m c}(t) \leq U B \quad \forall c, t \\
& N_{m c}(t-1)+n_{m c}^{+}(t)-n_{m c}^{-}(t)=N_{m c}(t) \quad \forall m, c \quad \forall t>1 \\
& \sum_{c=1}^{C}\left(n_{m c}^{+}(t)-n_{m c}^{-}(t)\right) \leq Y_{m}(t) \quad \forall m, t \\
& \sum_{c=1}^{C} N_{m c}(t) \geq \sum_{c=1}^{C} N_{m c}(t) \quad \forall m \quad \forall t>1 \\
& \sum_{m=1}^{M} \varepsilon_{j p m c}(t)=Z_{j p c}(t)\left(1-\delta_{p}(t)\right) \quad \forall j, p, c, t \\
& \sum_{c=1}^{C} Z_{j p c}(t)=1 \\
& \tilde{Q}_{p}(t) \leq D_{p}^{u}(t) \\
& \forall j, p, t \\
& \forall p, t \\
& \forall j, p, m, c, t \\
& W_{j p m c}(t)=\left(h_{j p m} \cdot Q_{p}(t) \cdot \varepsilon_{j p m c}(t)\right) / C_{m} \\
& \bar{W}_{j p c}(t)=\left(Z_{j p c}(t) \cdot \sum_{m=1}^{M} W_{j p m c}(t) \cdot N_{m c}(t)\right) /\left(\sum_{m=1}^{M} N_{m c}(t)\right) \quad \forall j, p, c, t \\
& 0 \leq \delta_{p}(t) \leq 1 \quad \forall p, t \\
& 0 \leq \varepsilon_{\text {jpmc }}(t) \leq 1 \\
& \forall j, p, m, c, t \\
& Z_{j p c}(t) \in\{0,1\} \\
& \forall j, p, c, t \\
& N_{m c}(t), n_{m c}^{+}, n_{m c}^{-}, Q_{p}(t) \geq 0 \text { \& Integer }
\end{aligned}
$$

\subsection{Model Defuzzification}

Since some parameters in form of variables and resources are uncertain and showed as fuzzy numbers, the proposed non-symmetric fuzzy model is converted into a crisp one by applying the $\alpha$ cut concept according to the Verdegay's approach [18]. It means that the minimum preference level determined by the DM is equal to $\alpha$. Therefore, we substitute the fuzzy demand for part $\mathrm{p}\left(Q_{p}\right)$, by crisp parameters, named $Q_{p}$, through defining appropriate $\alpha$-cut constraints (see Equations 18 to 20):

$$
\begin{aligned}
& \mu\left(D_{p}(t)\right) \geq \alpha \quad \forall p, t \\
& 0 \leq \alpha \leq 1
\end{aligned}
$$

Membership functions of Equation are substituted and then the following constraints are added to the primary model, which limit the production volume and indicate the confidence level of the DM. 
$Q_{p}(t) \geq \alpha \cdot D_{p}^{u}(t)+(1-\alpha) \cdot D_{p}^{l}(t) \quad \forall p, t$

\section{Proposed Multi-Objective Scatter Search}

In this section we propose a new approach to solve DCMS ${ }^{4}$ problem with two objective functions. As shown in the figure 2, scatter search algorithm has 5 phases to solve the single objective problem. Other procedures added or modified to produce Pareto optimal solutions by considering dynamic Pareto archive set, produce initial solution to produce better output from algorithm.

This method was first introduced by Glover [19] as a meta-heuristic method for integer programming. It was conceived as a relaxation, which was designed for the solution of integer programming problems, such as surrogate constraint relaxation. However, links between the approaches have increased in recent years. In the original SS proposal by Glover as a method that uses a succession of coordinated initializations to generate solutions. He introduced the reference set (RefSet) of solutions and several guidelines, including that the search takes place in a systematic way as oppose to the random designs of other methods (e.g., genetic algorithms). The approach was conceived to begin by identifying a convex combination of the reference points. This central point, together with subsets of the initial reference points, was then used to define new sub-regions. Thus, analogous central points of the sub-regions were examined in a logical sequence. Finally, these latter points were rounded (in a broad sense, depending on the solution representation) to obtain the desired solutions. These observations and principles lead to the well-known template consisting of five methods as follows:

1. Diversification generation method.

2. Improvement method.

3. Reference set update method.

4. Subset generation method.

5. Solution combination method.

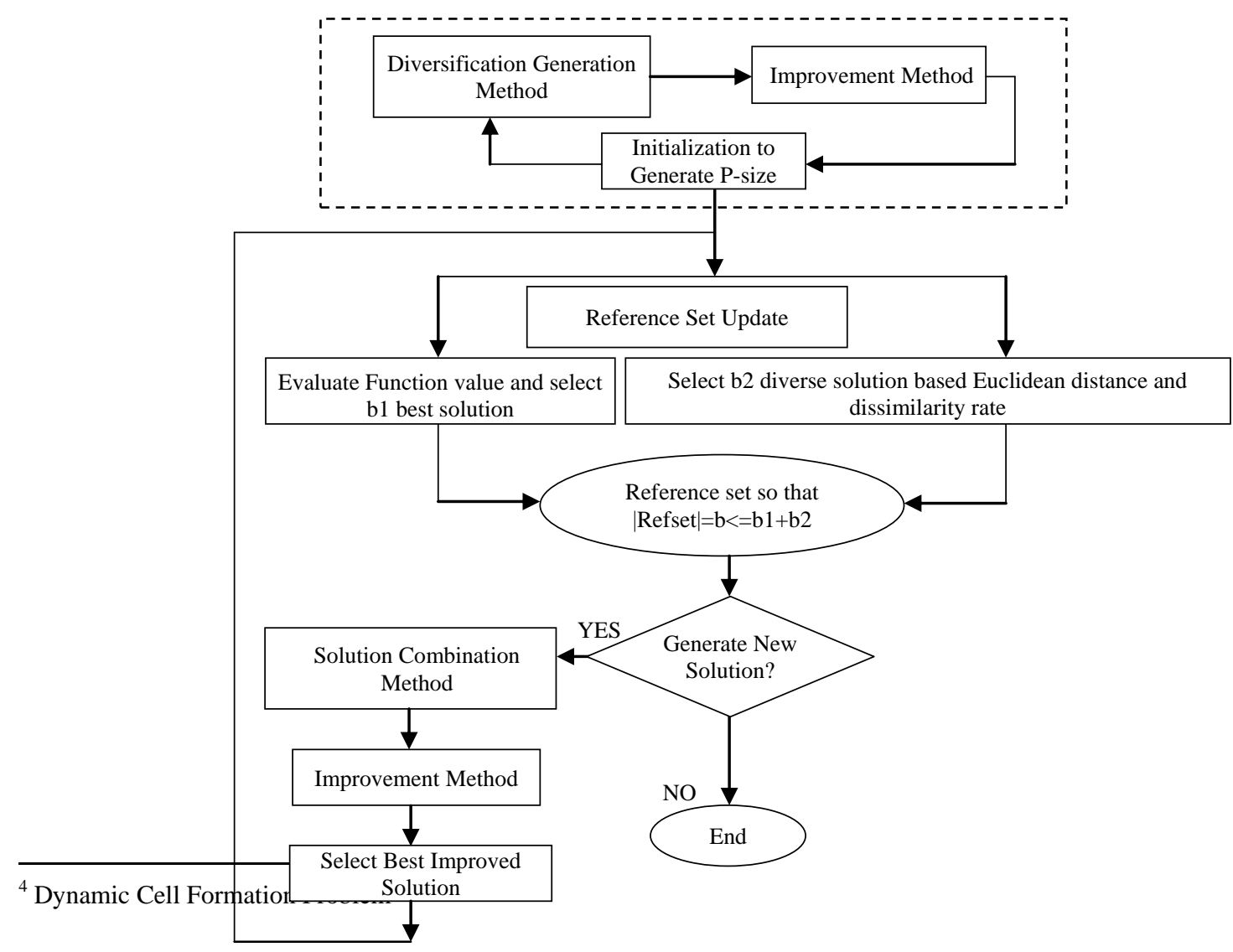


Figure 2. Flow chart of scatter search

\subsection{Tagouchi Design}

Taguchi method offers a systematic approach, simple and yet efficient optimized design parameters of each algorithm, using a limited set of experiments. Two main tools for change and the Taguchi method systematically test different levels of each factor, are: 1) design of experiments especially insyarra lanogohtro ; 2)N / S ratio.

\subsection{Planning}

In order to comprehensively evaluate all the parameters used; we define five levels of the parameters we studied. Also, since the type of crossing and mutation operators such factors in any evolutionary algorithm based on population and among them are the SS algorithm, so our four Crossover operator and mutation as the four levels studied these operators in terms take. The following table summarize, factors considered and their levels as the first stage results show that Taguchi method.

\subsection{Conducting}

We calculate Mean value and the S / $\mathrm{N}$ ratio to each of two criteria $\mathrm{f} 1$ and $\mathrm{f} 2$ separately based on the best solution produced by algorithm MOSS. This is taken from there because we compared the resolutions of both criteria $\mathrm{f} 1$ and $\mathrm{f} 2$ at the same time we have considered, so the algorithms adjust the parameters of the policy can be used.

Table 2. Effective factors of MOSS and each levels

\begin{tabular}{ccccc}
\hline & & & Factors & \\
\hline Levels & Cross Rate $\in[0,1]$ & b1/b2 & Mutation Type & Crossover Type \\
\hline 1 & 0 & 0.5 & 2 & 2 \\
2 & 0.25 & 0.75 & 3 & 3 \\
3 & 0.5 & 1.25 & 4 & 3 \\
4 & 0.75 & 1.75 & 2 & 3
\end{tabular}

\subsection{Analysis}

When all the $\mathrm{S} / \mathrm{N}$ ratios and the average response to each of the tests were calculated, Taguchi method approaches of a chart to analyze the data uses. In this approach, plots the average ratios $\mathrm{S} / \mathrm{N}$ and the average mean response for each factor and to exchange their different levels, are plotted. 

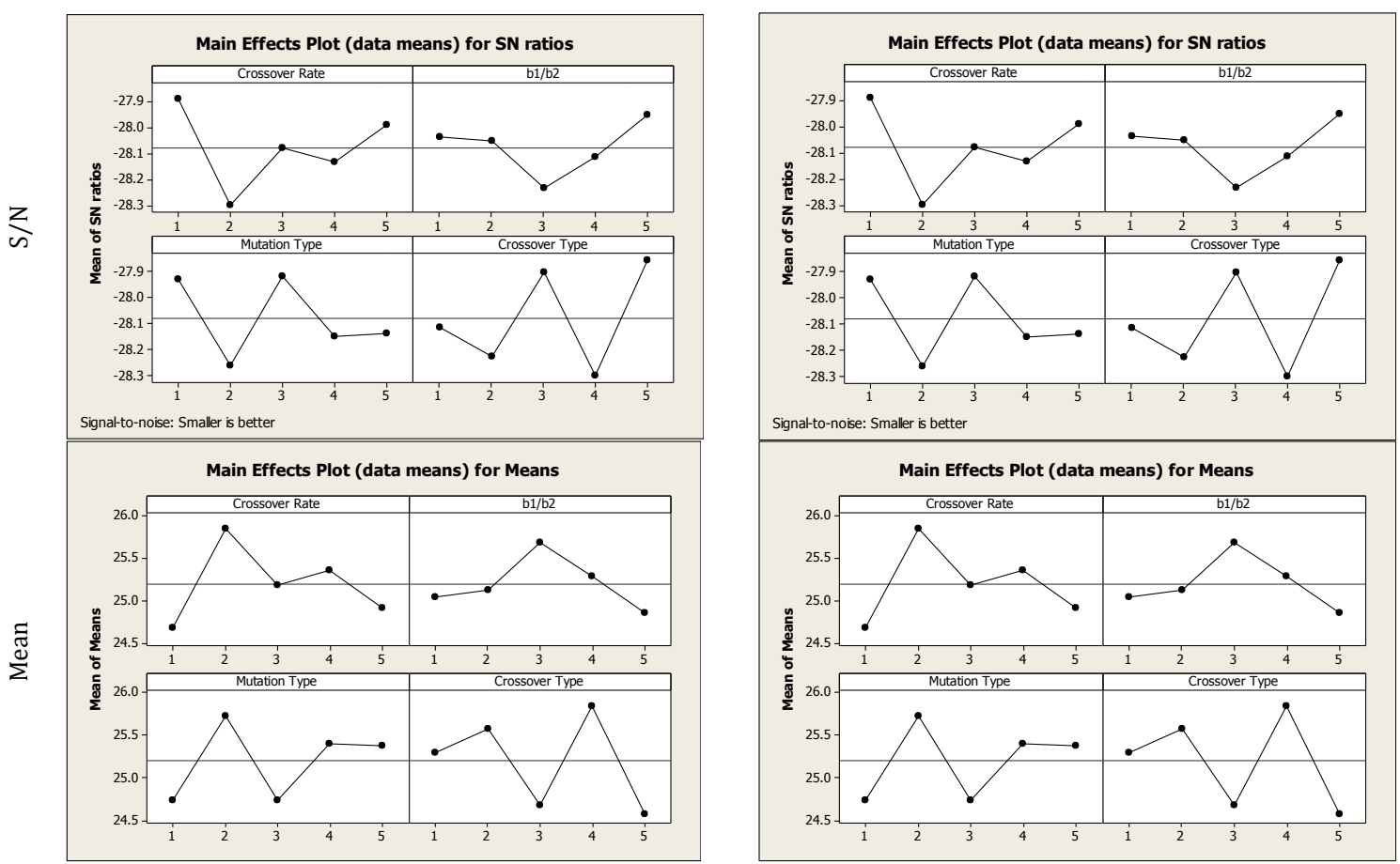

Figure 3. Taguchi design for making MOSS robust

\section{Computational results}

In this section, performance model for manufacturing cell formation problem studied by a few issues and all matters placed by the software MATLAB version 7.6 has been implemented. The experiments on a Laptop and GHZ 2.66 MB 512 RAM Windows XP operating system are done. Each test problem is shown by the code M-P-O-C-T which $\mathrm{M}$ indicates the number in front of the machine count, the number of $P$ is represented parts count; $O$ represents the number of digits after the operation of each piece, after the figure represents the number of cells allowed $\mathrm{C}$ and $\mathrm{T}$ is the number of periods.

Table 3. NSGA-II parameters

\begin{tabular}{|c|c|c|c|c|}
\hline population & No. of Generation & Cross over rate & Mutation Rate & Archive Size \\
\hline 100 & 200 & 0.95 & 0.05 & 30 \\
\hline
\end{tabular}

Information about 10 samples is randomly generated. To compare the results of the proposed algorithm, a number of quality measures relating specially for multi-objective function which are widely used in the literature have been applied. The following description explains these measures.

Table 4. MOSS parameters

\begin{tabular}{|c|c|c|c|c|c|c|c|c|}
\hline Population & $\begin{array}{c}\text { No. of } \\
\text { Generation }\end{array}$ & $b_{2}$ & $b_{1}$ & $\begin{array}{c}\text { No. of } \\
\text { Iteration } \\
\text { without } \\
\text { Changing }\end{array}$ & $\begin{array}{c}\text { Local } \\
\text { Iteration } \\
\text { Number }\end{array}$ & $\begin{array}{c}\text { Cross Over } \\
\text { Rate }\end{array}$ & $\begin{array}{c}\text { Mutation } \\
\text { Rate }\end{array}$ & Archive Size \\
\hline 100 & 200 & 15 & 20 & 3 & 5 & 0.75 & 0.05 & 30 \\
\hline
\end{tabular}

\subsection{Hyper Area Ratio Metric}

This index shows the number of optimal solutions obtained by each algorithm. Since the number of Pareto optimal solutions is not clear for us, requires that by Comprehensive enumeration the total optimal solutions have to be obtained for each test problem. But since obtaining the non-dominated optimal solutions by comprehensive enumeration is timeconsuming task even for small scale problems, we use a way to calculate this measure for each 
test problems after determination of optimal solutions of each test problem and comparing its solutions to the dominant optimal solutions of each algorithm, number of non-dominated optimal solutions can be realized.

The metric definition is the following:

$H A R M=\frac{H_{e s}}{H_{s c}}$

Where $H_{e s}$ the area is occupied by the solution set of practical Pareto front, and $H_{s c}$ is the area occupied by the theoretical Pareto front. As finding the theoretical Pareto front is practically impossible, we produce it by all non-dominated solutions provided from three algorithms. If the value of the hyper area ratio is smaller than 1 , the points of the practical tradeoff surface are not spread over the whole tradeoff surface. If the value of the hyper area ratio is greater than 1 , the practical tradeoff surface is distant from the tradeoff surface. [20]

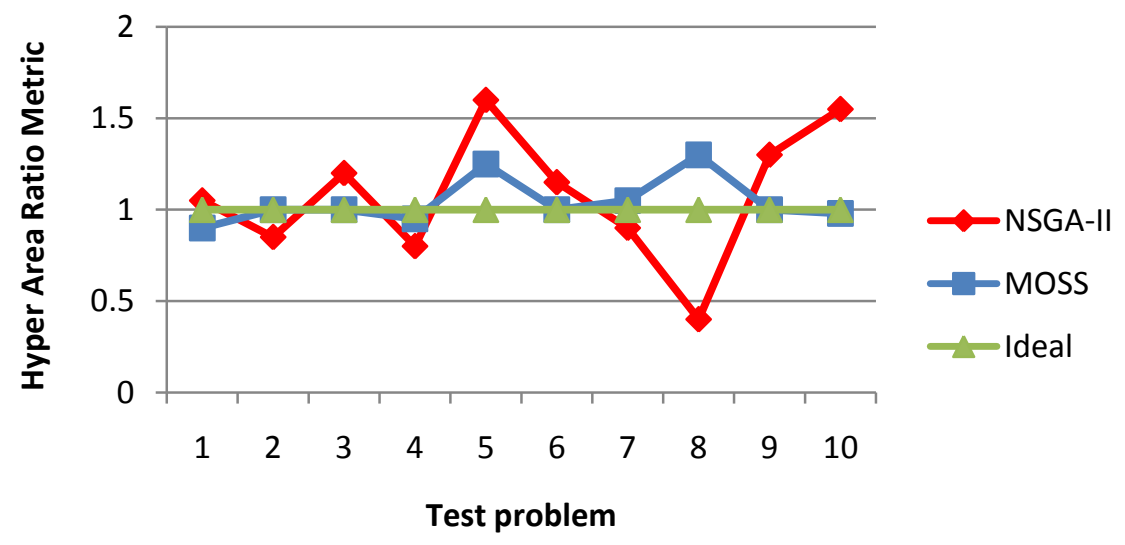

Figure 4. Hyper area ratio metric for each algorithms

\subsection{Spacing metrics}

Spacing metrics can be classified in the diagnosis of uniformity in distribution of answers to help us Pareto curve. This index is calculated as follows.

$$
\begin{aligned}
& d i=\min _{j}\left(\left|f_{1}^{i}(\vec{x})-f_{1}^{j}(\vec{x})\right|+\left|f_{2}^{i}(\vec{x})-f_{2}^{j}(\vec{x})\right|\right) \\
& S=\left[\frac{1}{n-1} \cdot \sum_{i=1}^{n}\left(\bar{d}-d_{i}\right)^{2}\right]^{\frac{1}{2}} \quad j=1,2, \ldots, n, i \neq j
\end{aligned}
$$

$\bar{d}$ is the mean value of $d_{i}$ and $\mathrm{n}$ is the number of Pareto curve's elements. At First, for each Pareto point related $d_{i}$ is calculated. To calculate this parameter, the nearest neighbor points are used. Then $\mathrm{S}$ is calculated which is classified as spacing metric. The value of this index is closer to zero indicates more and better distribution of answers. Basic definitions related to this index by Collette and Siarry is expressed. [20] 


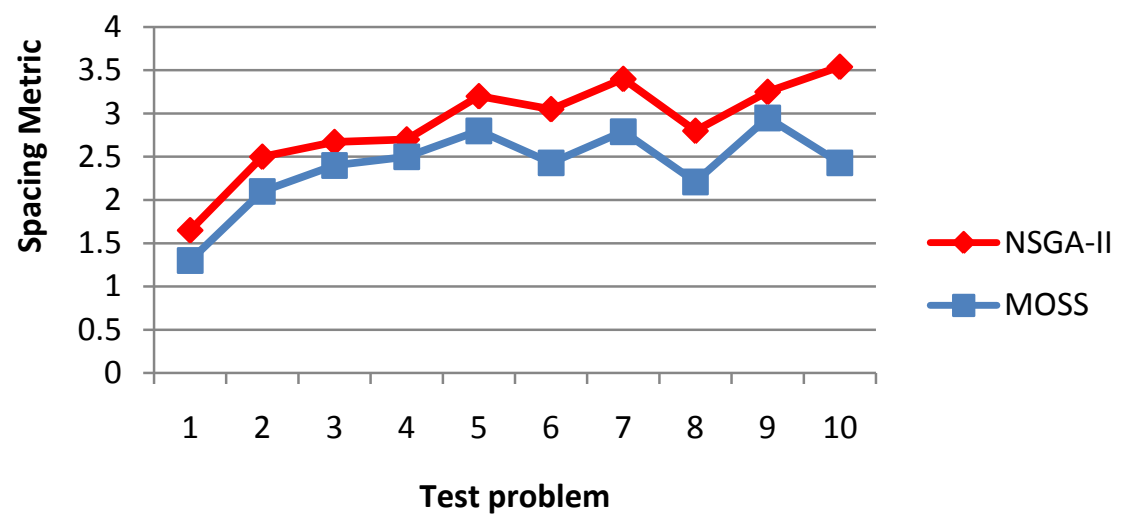

\subsection{Quality metrics}

Figure 5. Spacing metric for each algorithm

Number of Pareto solutions index alone cannot appropriate metrics to evaluate the two because algorithms is not clear how many of the answers provided by a Pareto algorithms are with high quality. Quality metrics is calculated by putting together dominant response algorithm produced both $\mathrm{A}$ and $\mathrm{B}$ and such ratio in the number of share each method of final Pareto solutions.

An experiment for studying the influence of two methods and ten objective spaces (test problems) on the spacing metric with ten replicates is performed. The analysis of variance is shown in Table 4. The main effects and interaction are significant. MOSS provides lower value of the spacing metric.[20]

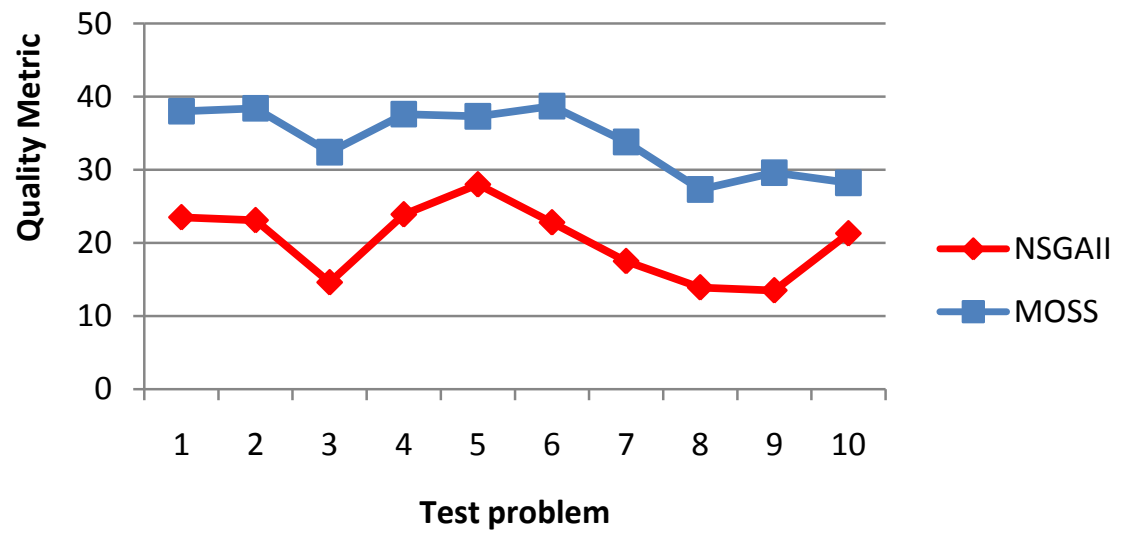

Figure 6. Quality metric for each algorithms

Total information about 10 test problems based on runtime, hyper area ration metric, quality metric and spacing metric are shown in Table 5. It is worthy nothing that each of algorithm 10 times runs and average of results provided as illustrated in the Table 6.

Hyper Area Ratio Metric come in many test problems by MOSS algorithm compared with NSGAII algorithm shows that the standard deviation is closer to number 1.

Number of solutions generated by the two dominant algorithms in a way that all the problems generated by the number of solutions to MOSS algorithm NSGA-II, consistently show higher values and this indicates that this is a good algorithm MOSS has dominated in finding solutions. In the spacing metric column between the classifications, distribution more efficient solutions can be generated by the proposed algorithm is concluded.

Table 5. ANOVA regarding to quality metric

\begin{tabular}{llllllll}
\hline Source of Variation & SS & df & MS & F & P-value & F crit \\
\hline
\end{tabular}




\begin{tabular}{lllllll}
\hline Problems & 1775.54 & 9.00 & 197.28 & 142.84 & 0.00 & 2.00 \\
Algorithms & 3994.24 & 1.00 & 3994.24 & 2891.97 & 0.00 & 3.96 \\
Interaction & 246.44 & 9.00 & 27.38 & 19.83 & 0.00 & 2.00 \\
Within & 110.49 & 80.00 & 1.38 & & & \\
Total & 6126.71 & 99.00 & & & &
\end{tabular}

The time needed for implementation show the two algorithms. Could be concluded that the proposed algorithm has a mechanism Because of the possible search space will solve, obviously more time than NSGA-II algorithm can be spent. Yet the quality of answers to MOSS algorithm NSGA-II is to increase a runtime algorithm to solve the NSGA-II is negligible.

Table 6. result of MOSS and NSGA-II

\begin{tabular}{ccccccccc}
\hline \multirow{2}{*}{$\begin{array}{c}\text { Test } \\
\text { Problem }\end{array}$} & M-P-O-C-T & \multicolumn{2}{c}{ Runtime } & \multicolumn{2}{c}{ HARM $^{\varsigma}$} & \multicolumn{2}{c}{ QM } & \multicolumn{2}{c}{ SM $^{\triangleright}$} \\
\cline { 2 - 9 } & MOSS & NSGA-II & MOSS & NSGA-II & MOSS : NSGA-II & MOSS & NSGA-II \\
\hline 1 & $3-4-2-2-2$ & 110.36 & 89.43 & 0.9 & 1.05 & $38: 235$ & 1.3 & 1.65 \\
2 & $3-4-2-2-2$ & 119.46 & 93.56 & 1 & 0.85 & $38.4: 23$ & 2.1 & 2.5 \\
3 & $4-3-3-2-2$ & 100.95 & 88.12 & 1 & 1.2 & $32.4: 14.6$ & 2.4 & 2.67 \\
4 & $6-5-3-2-2$ & 105.84 & 85.35 & 0.95 & 0.8 & $37.6: 23.9$ & 2.5 & 2.7 \\
5 & $5-6-3-3-2$ & 130.2 & 88.56 & 1.25 & 1.6 & $37.3: 29$ & 2.8 & 3.2 \\
6 & $7-5-3-3-2$ & 125.32 & 90.04 & 1 & 1.15 & $38.7: 22.8$ & 2.43 & 3.05 \\
7 & $7-6-4-3-2$ & 109.05 & 83.31 & 1.05 & 0.9 & $33.8: 17.5$ & 2.79 & 3.4 \\
8 & $7-6-3-3-2$ & 145.09 & 120.32 & 1.3 & 0.4 & $27.3: 13.9$ & 2.21 & 2.8 \\
9 & $8-6-4-3-3$ & 168.80 & 140.50 & 1 & 1.3 & $29.6: 13.5$ & 2.95 & 3.25 \\
10 & $8-6-4-4-3$ & 158.44 & 150.45 & 0.98 & 1.55 & $28.2: 21.3$ & 2.43 & 3.54
\end{tabular}

\section{Conclusion}

In this paper a new multi-objective dynamic cell formation considering fuzzy demand was proposed. Due to the NP-hardness nature of our problem we designed a multi-objective scatter search for finding Pareto optimal solution of mentioned test problems. To illustrate the performance of MOSS, we benchmarked the performance of our proposed algorithm with one of the most popular and well-known algorithm which is widely used for solving multi-objective problem called NSGA-II base on some quality measures which are introduced in the literature.

Results from implementing two algorithms on test problems show that proposed algorithm has the superior output comparing NSGA-II algorithm. It can be concluded, implementing proposed algorithm will have promising results for solving multi-objective problems.

\section{Acknowledgement}

This study was partially supported by the Islamic Azad University- Nowshahr Branch. The authors would like to acknowledge for this financial support.

\section{Reference}

1. Vakharia, A. J. and Kaku, B. K., "Redesigning a cellular manufacturing system to handle long-term demand changes: A methodology and investigation", Decision Sciences, Vol. 24, No. 5, (1993), 909-929.

2. Harhalaks, G., Nagi, R. and Proth, J., "An efficient heuristic in manufacturing cell formation to group technology applications”, Int. Journal of Production Research, Vol. 28, No. 1, (1990), 185-198.

\footnotetext{
${ }^{5}$ Spacing Metric

${ }^{6}$ Hyper Area Ratio Metric
} 
3. Wilhelm, W., Chiou, C. and Chang, D., "Integrating design and planning considerations in cellular manufacturing”, Annals of Operation Research, Vol. 77, No. 1, (1998), 97-107.

4. Askin, R. G., Selim, H. M. and Vakharia, A. J., "A methodology for designing flexible cellular manufacturing systems”, IIE Transactions, Vol. 29, (1997), 599-610.

5. Chen, M., "A mathematical programming model for systems reconfiguration in a dynamic cell formation condition", Annals of Operations Research, Vol. 77, No. 1, (1998), 109-128.

6. Balakrishnan, J. and Cheng, C. H., "Dynamic cellular manufacturing under multi-period planning horizons", Journal of Manufacturing Technology Management, Vol. 16, No. 5, (2005), 516-530.

7. Tavakkoli-Moghaddam, R. and Safaei, N., "A generalized dynamic cell formation problem with fuzzy demand and unreliable facilities", Proceeding of the 3rd International Conference on Group Technology/Cellular Manufacturing, Groningen, Netherlands, (2006), 349-356.

8. Defersha, F. M. and Chen, M., "A comprehensive mathematical model for the design of cellular manufacturing system”, International Journal of Production Economics, Vol. 103, (2006), 767-783.

9. Safaei, N., Saidi-Mehrabad, M., Tavakkoli-Moghaddam, R. and Sassani, F., "A fuzzy programming approach to a cell formation problem with dynamic and uncertain conditions", Fuzzy Sets and Systems, Vol. 159, No. 2, (2008), 215-236.

10. Torabi, S. A. and Hassini, E., "An interactive possibilistic programming approach for multiple objective supply chain master planning", Fuzzy Sets and Systems, Vol. 159, No. 2, (2008), 193-214.

11. Shankar, R. and Vrat, P., "Some design issues in cellular manufacturing using the fuzzy programming approach", International Journal of Production Research, Vol. 37, No. 11, (1999), 2545-2563.

12. Seifoddini, H., "A probabilistic model for machine cell formation", Journal of Manufacturing Systems, Vol. 9, No. 1, (1990), 69-75.

13. Tavakkoli-Moghaddam, R., Safaei, N. and Babakhani, M., "Solving a dynamic cell formation problem with machine cost and alternative process plan by memetic algorithms", Lecture Notes in Computer Science, Vol. 3777, (2005b), 213-227.

14. Tavakkoli-Moghaddam, R., Aryanezhad, M. B., Safaei, N., Vasei, M. and Azaron, A., "A new approach for the cellular manufacturing problem in fuzzy dynamic conditions by a genetic algorithm", Journal of Intelligent and Fuzzy Systems, Vol. 18, No. 4, (2007), 363-376.

15. Tavakkoli-Moghaddam, R. and Safaei, N., "A generalized dynamic cell formation problem with fuzzy demand and unreliable facilities", Proceeding of the 3rd International Conference on Group Technology/Cellular Manufacturing, Groningen, Netherlands, (2006), 349-356.

16. Tanaka, H., Okuda, T. and Asai, K., "On fuzzy mathematical programming”, Journal of Cybernetics, Vol. 3, (1974), 37-46.

17. Lai, Y. J. and Hwang, C. L., "Fuzzy mathematical programming: methods and applications", SpringerVerlag, Berlin (1992).

18. Verdegay, J. L., Fuzzy mathematical programming, in: M.M. Gupta and E. Sanchez (Eds.), "Fuzzy information and decision processes", North-Holland, Amsterdam (1982), 231-237.

19. Glover, F. (1977). Heuristics for integer programming using surrogate constraints. Decision Sciences, 8(1), 156-166.

20. ColletteY, Siarry P. Multi-objective optimization: principles and case studies. NewYork: Springer; 2003. 